\title{
DESCRIPCIÓN DE UN NUEVO TRECHUS CLAIRVILLE, 1806 (COLEOPTERA, CARABIDAE, TRECHINI) DE LOS MONTES CANTÁBRICOS ORIENTALES (NORTE DE ESPAÑA)*
}

\author{
V. M. Ortuño** y M. Toribio***
}

\begin{abstract}
RESUMEN
Se describe Trechus pisuenensis sp. nov. de los montes cantábricos orientales. Se estudia su morfología y, en particular, la genitalia masculina y femenina. Asimismo, se discute su afinidad con aquellas especies más próximas (grupo T. angusticollis o grupo T. amplicollis ?). También se aportan datos sobre su biología y ecología.

Palabras clave: Taxonomía, Coleoptera, Carabidae, Trechini, Península Ibérica, Trechus pisuenensis sp. nov.
\end{abstract}

\begin{abstract}
Description of a new Trechus Clairville, 1806 (Coleoptera, Carabidae, Trechini) from the eastern Cantabrian Mountains (northern Spain)

Trechus pisuenensis sp. nov. is described. Its morphology is examined and, in particular, the structure of the male and female genitalia. Affinities with closely related species (T. angusticollis group or T. amplicollis group ?) are discussed. Biological and ecological data are also included.

Key words: Taxonomy, Coleoptera, Carabidae, Trechini, Iberian Peninsula, Trechus pisuenensis sp. nov.
\end{abstract}

\section{Introducción}

Trechus Clairville, 1806 es un amplio género que cuenta con más de 500 especies (Casale et al., 1998). Hasta la fecha se conoce de la región Holártica y el este de África. En la Península Ibérica se han citado 49 especies (Serrano, 2003) muchas de las cuales son endémicas. La sistemática interna de este género es compleja, razón por la cual se propusieron "grupos de especies" (Jeannel,
1927) que han facilitado ulteriores trabajos taxonómicos y, a la par, han orientando sobre la posible afinidad de sus especies. El conjunto de los Trechus ibéricos se reúnen en nueve grupos (sensu Jeannel, 1927), si bien desde su propuesta se han visto incrementados notablemente en número de especies. Actualmente el elenco ibérico de Trechus queda distribuido de forma desigual en los siguientes grupos: "grupo T. angusticollis" con 12 especies, "grupo T. quadristriatus" con 6 especies, "grupo $T$.

\footnotetext{
* Trabajo financiado por el Ministerio español de Ciencia y Tecnología dentro de programa "Ramón y Cajal” y por el proyecto UAH-PI2004/026 de la Universidad de Alcalá.

** Departamento de Zoología y Antropología Física. Facultad de Biología. Universidad de Alcalá. E-28871 - Alcalá de Henares. Madrid (España). vicente.ortuno@uah.es

*** Avda. Viñuelas, 32. E-28760 - Tres Cantos. Madrid (España). marcostoribio@telefonica.net
} 
fulvus" con 9 especies, "grupo T. uhagoni" con 11 especies, "grupo T. pandellei" con 1 especie, "grupo T. pyrenaeus" con 5 especies, "grupo T. tingitanus" con 1 especie, "grupo T. distigma" con 2 especies y "grupo T. austriacus" con 2 especies.

En el presente artículo damos a conocer una especie nueva de Trechus procedente de los montes cantábricos orientales, cuya peculiar morfología dificulta su integración en los grupos de especies conocidos para la Península Ibérica. En este trabajo procedemos a la descripción formal de la especie, aportamos datos sobre su biología y discutimos su posible asignación al "grupo T. angusticollis" o al "grupo T. amplicollis", éste último sin representación en Iberia.

\section{Trechus pisuenensis sp. nov.}

(Figs. 1-8)

Holotipo: Cantabria: Aloños (Hayal de Aloños), 19-VIII2004, $1 \sigma^{7}$ (V.M. Ortuño leg.), depositado en la colección del Departamento de Zoología y Antropología Física (DZAF-UA), Universidad de Alcalá, Alcalá de Henares (Madrid. España).

PARatipos: Cantabria: Aloños (Hayal de Aloños), 19-VIII2004, $120^{7} 0^{7}$ y 7 우 우 (V.M. Ortuño y M. Toribio leg.), 2 ejemplares depositados en el Museo Nacional de Ciencias Naturales ( $n^{\circ}$ de referencia: MNCN-11595), CSIC, Madrid y el resto en las colecciones del DZAF-UA y M. Toribio.

DiAGNOSIS: Insecto braquíptero y microftalmo. Tegumento despigmentado (testáceo) más o menos brillante con microrreticulación poco aparente. Pronoto trapezoidal, apenas transverso, con los lados levemente sinuados por delante de los ángulos posteriores que son rectos y vivos. Élitros subparalelos, con los hombros muy acusados; todas las estrías profundas y bien marcadas hasta casi el ápice. Protarsos de los machos con los dos primeros tarsómeros dilatados. Lóbulo medio del edeago recto, con la base y el ápice ventralmente curvados; lámina apical aguzada; saco interno con una pieza esclerotizada de aspecto laminar y alabeado, de tal modo que puede parecer que existe una segunda pieza más pequeña y corniforme.

DESCRIPCIÓN: Longitud del holotipo (desde las mandíbulas al ápice de los élitros): $4,8 \mathrm{~mm}$. Longitud de los paratipos: 3,9-4,8 $\mathrm{mm}$.

Cabeza casi tan larga (sin contar las mandíbulas) como ancha y manifiestamente más estrecha que el pronoto; mandíbulas, piezas labiales y maxilares típicas del género; diente labial levemente escotado; surcos frontales profundos que se prolongan hasta el clípeo, bordeando el área ocular; sienes largas (al menos dos veces más largas

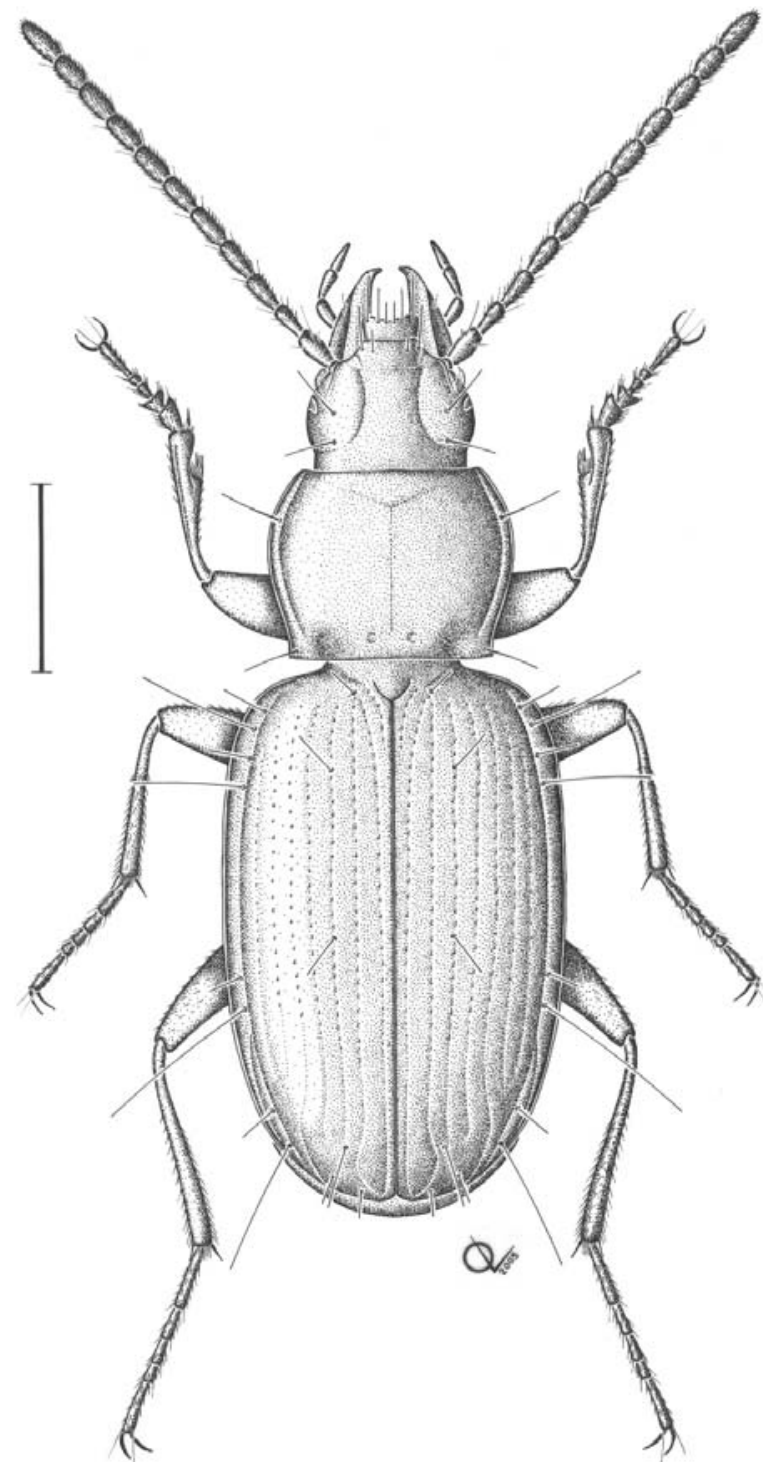

Fig. 1.- Hábitus de Trechus pisuenensis sp. nov. Holotipo. Escala: $1 \mathrm{~mm}$.

Fig. 1.- Habitus of Trechus pisuenensis sp. nov. Holotype. Scale bar: $1 \mathrm{~mm}$.

que los ojos), muy convexas, con pilosidad diminuta; ojos pequeños nada prominentes ni convexos, éstos reducidos a unos pocos ommatidios; dos setas supraoculares de las que, la anterior se sitúa casi al nivel del borde posterior del ojo y la posterior muy alejada del mismo. Antenas largas y, en general, con los antenómeros casi tres veces tan largos como anchos. 


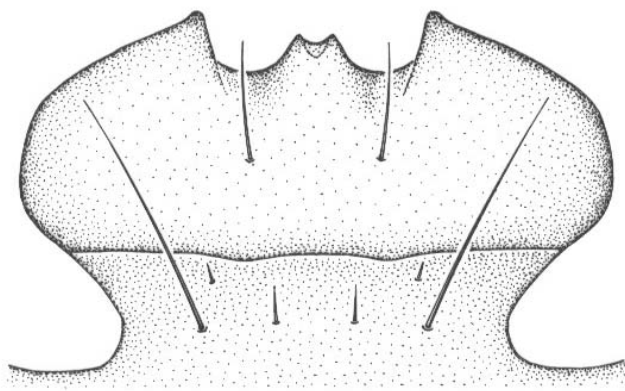

Fig. 2.- Labio y pieza prebasilar de Trechus pisuenensis sp. nov. Paratipo. Escala: $0,2 \mathrm{~mm}$.

Fig. 2.- Labium and prebasilar piece of Trechus pisuenensis sp. nov. Paratype. Scale bar: $0.2 \mathrm{~mm}$.

Pronoto apenas convexo, ligeramente transverso (un poco más ancho que largo), con su mayor anchura por delante del medio; base recta, no saliente y tan ancha o más ancha que el borde anterior; borde lateral levemente sinuado en las proximidades de los ángulos posteriores, éstos rectos y vivos; canal lateral ancho y profundo en toda su longitud; surco medio bien indicado; dos setas pronotales con carácter bilateral (la anterior situada en el primer tercio y la posterior ligeramente por delante del ángulo); fosetas basales anchas, poco profundas y no punteadas.

Élitros anchos, manifiestamente subparalelos que alcanzan su anchura mayor en el centro; hombros redondeados pero conspicuos; borde basal poco inclinado que acaba cerca del origen de la $5^{\mathrm{a}}$ estría; estrías profundas y fuertemente punteadas en toda su longitud (la $7^{\mathrm{a}}$, puede mostrarse algo más superficial); $8^{\mathrm{a}}$ estría muy separada del margen; estriola apical recurrente profunda, bien indicada y unida con la terminación de la $5^{\mathrm{a}}$ estría; interestrías ligeramente convexas; canal lateral ancho; seta discal anterior situada en el quinto basal (o ligeramente adelantada) y seta discal posterior casi en el centro de los élitros, ambas sobre la $3^{\mathrm{a}}$ estría; poro escutelar en el origen de la $1^{\text {a }}$ estría; serie umbilical típica del género, con cuatro setas equidistantes en el grupo humeral y cuatro setas en el grupo apical (dos anteriores y dos posteriores).

Patas normales, con las tibias anteriores surcadas longitudinalmente en el margen externo del dorso;

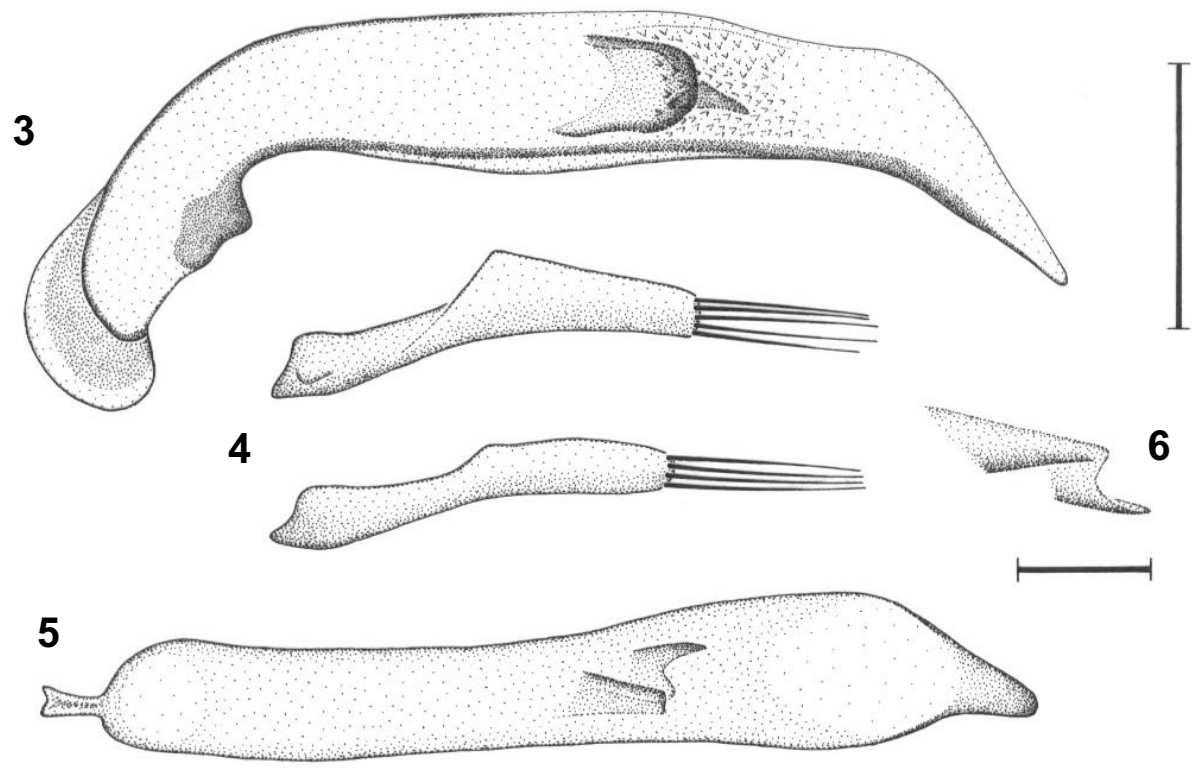

Figs. 3-6.- Genitalia masculina de Trechus pisuenensis sp. nov. Holotipo. 3: Lóbulo medio en visión lateral izquierda. 4: Parámeros en visión lateral izquierda. 5: Lóbulo medio en visión dorsal. 6: Pieza interna del lóbulo medio en visión ventral. Escala (3-5): 0,3 mm. Escala (6): 0,1 $\mathrm{mm}$.

Figs. 3-6.- Male genitalia of Trechus pisuenensis sp. nov. Holotype. 3: Median lobe in left lateral view. 4: Parameres in left lateral view. 5: Median lobe in dorsal view. 6: Inner piece of median lobe in ventral view. Scale bar (3-5): $0.3 \mathrm{~mm}$. Scale bar (6): $0.1 \mathrm{~mm}$. 

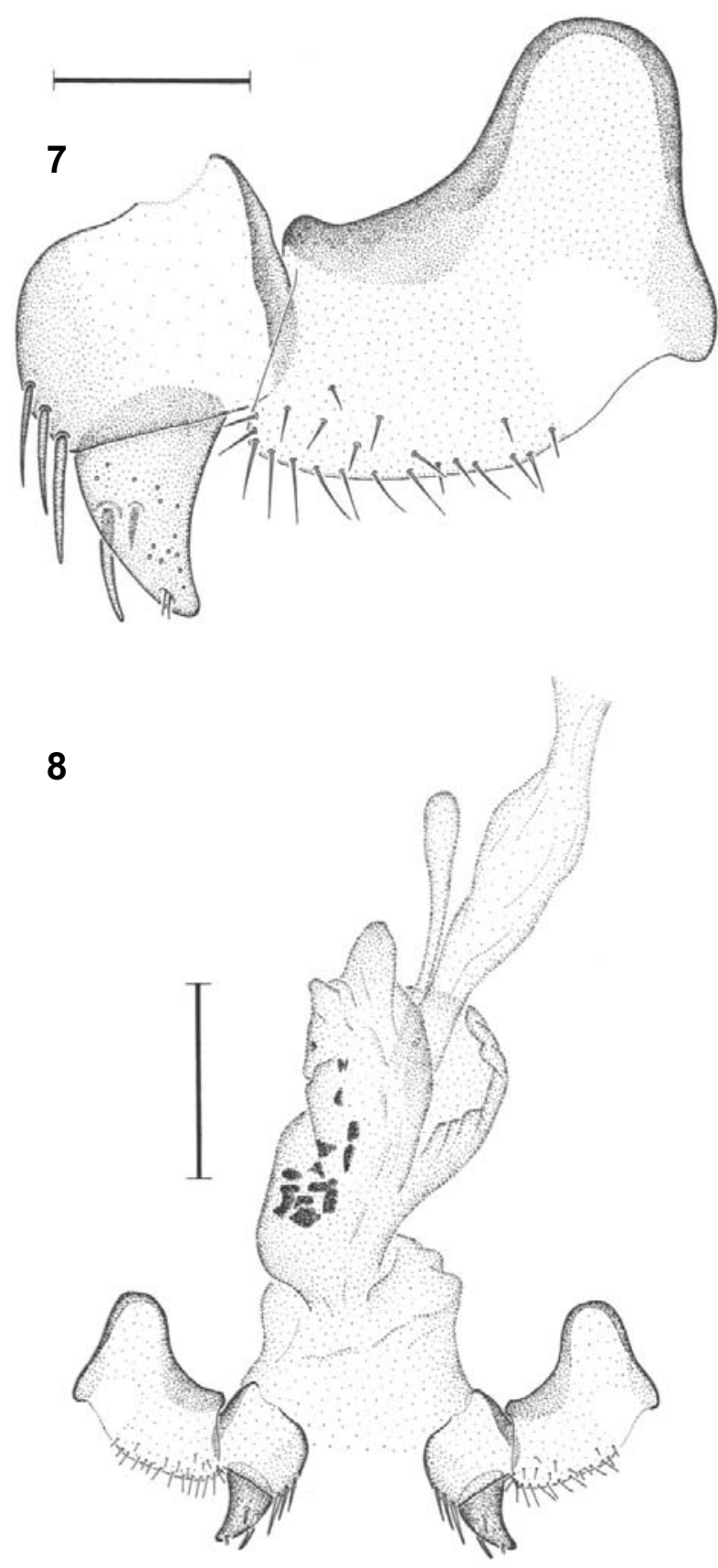

Figs. 7-8.- Genitalia femenina de Trechus pisuenensis sp. nov. en visión ventral. Paratipo. 7: Detalle de la genitalia externa izquierda (= armadura genital). 8: Genitalia externa e interna. Escala (7): 0,1 mm. Escala (8): 0,3 mm.

Figs. 7-8.- Female genitalia of Trechus pisuenensis sp. nov. in ventral view. Paratype. 7: Detail of left external genitalia (= genital shield). 8: External and internal genitalia. Scale bar (7): $0.1 \mathrm{~mm}$. Scale bar (8): $0.3 \mathrm{~mm}$. los dos primeros protarsos de los machos claramente dilatados y dentados en el margen interno.

Edeago con el lóbulo medio alargado, rectilíneo y con la superficie ventral carenada longitudinalmente y un poco dilatada; bulbo basal arqueado en curva regular (forma un ángulo obtuso) y que porta en el extremo un gran alerón sagital; ápice bruscamente inclinado hacia la parte ventral, justo desde el comienzo del orificio apical, describiendo un plano inclinado; lámina apical aguzada tanto en visión lateral como dorsal. Parámeros anchos, subiguales (el izquierdo es apenas más largo que el derecho) y con cuatro o cinco setas apicales cada uno. Saco interno (visión lateral izquierda) con una pieza laminar en forma de media luna y la zona convexa orientada hacia el lado derecho; por detrás asoma otra lámina pequeña, con forma de diente agudo que apunta hacia el ápice del lóbulo medio; en visión ventral o dorsal se observa que no consiste en estar formado por dos piezas y que en realidad se trata de una sola lámina alabeada.

Genitalia externa (femenina) formada por los gonópodos IX que son dímeros (gonocoxito y gonosubcoxito) y los lateroterguitos IX; gonocoxito unguiforme con 2 setas espiniformes, en ocasiones 3 setas, que se insertan en la superficie dorsal cerca del margen interno; cerca del ápice se observa una pequeña foseta que aloja dos pequeñas setas sensoriales; gonosubcoxito tan largo como ancho, con tres gruesas setas espiniformes alojadas en el margen interno; lateroterguito IX aliforme, ligeramente esclerotizado con excepción de los márgenes anterior y externo; su setación se distribuye en dos grupos, uno junto al margen basal y constituido por una veintena de setas y un segundo grupo, de disposición más interna, formado por media docena de setas aproximadamente. Genitalia interna (femenina) mayoritariamente membranosa; la vagina es corta y ancha, y da paso a la bursa copulatrix, larga y profundamente invaginada antero-ventralmente, lo que le confiere cierto aspecto auricular; en la superficie ventral la bursa muestra esclerotizaciones a modo de pequeñas escamas; el oviducto impar se abre en el fondo de la invaginación de la bursa; junto a la base de éste aparece la espermateca que es alargada y asoma por la parte anterior de la bursa, haciéndose visible sólo los dos tercios distales (el último de diámetro algo mayor).

VARIABILIDAD: Entre los 20 especímenes conocidos se ha observado escasa variabilidad morfológica, que afecta fundamentalmente al tamaño; los especímenes de mayor y menor tamaño difieren en casi $1 \mathrm{~mm}$, lo que supone que los individuos más 


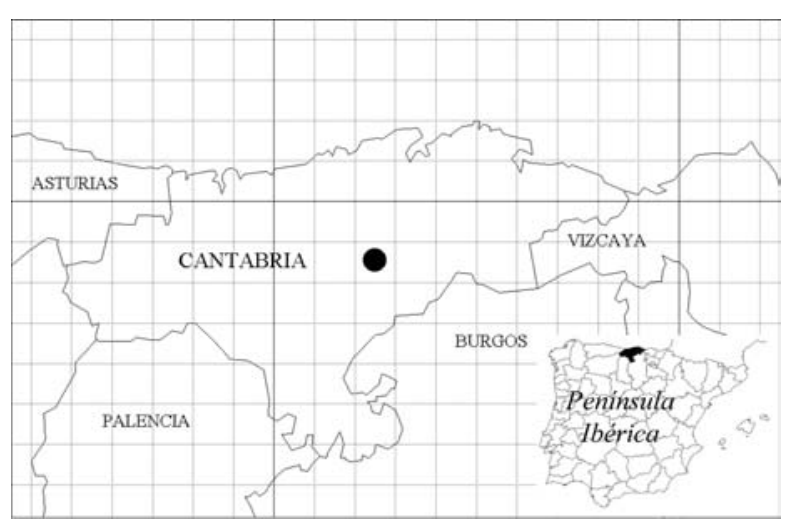

Fig. 9.- Mapa de localización de Trechus pisuenensis sp. nov. Retículo de UTM de $10 \times 10 \mathrm{~km}$.

Fig. 9.- Map of Trechus pisuenensis sp. nov. localization. Map grid (UTM $10 \times 10 \mathrm{~km}$ ).

pequeños lo son en un $19 \%$ de la talla de los mayores. También hay ligera variabilidad en la expresión del carácter "transversalidad del pronoto". Parece que en ambos casos estas singularidades no están vinculadas al sexo. Además, los gonocoxitos pueden estar asimétricamente setulados por la aparición de una seta espiniforme supernumeraria, característica que ya ha sido observada en otras especies de Trechini (Salgado \& Ortuño, 1998).

Etimología: El epíteto específico proviene de la latinización del nombre del valle que recoge las aguas de escorrentía (a las que parece vinculada $\mathrm{T}$. pisuenensis sp. nov.) del "Hayal de Aloños", nos referimos al valle del río Pisueña.

HÁBITAT y Biología: T. pisuenensis sp. nov., por el momento, sólo es conocido de la comarca del río Pisueña que se halla casi en el centro de Cantabria, demarcación territorial que limita al sur con los relieves más orientales de la Cordillera Cantábrica (macizo de Castro Valnera con su cota máxima de $1.700 \mathrm{~m}$ ), y al norte con los relieves prelitorales cuyas altitudes no superan los $600 \mathrm{~m}$. Este carábido nuevo queda confinado al área más montuosa conocida como "cuenca alta del río Pisueña". En esta zona se encuentra una pequeña "isla nemoral" conocida bajo el nombre de "Hayal de Aloños" (Fig. 9), localidad típica de T. pisuenensis sp. nov. El citado bosque se extiende entre los 500 y $800 \mathrm{~m}$ de altitud, ocupando la ladera NE del cordal que separa los valles del río Pas y del Pisueña, en donde se alza el Pico Tablao $(850 \mathrm{~m})$. El sustrato calizo sobre el que medra el bosque se muestra pendiente y quebrado por la cabecera del río Junquera y sus torrentes tributarios. Las condiciones del suelo son de cierta permeabilidad, por ello la escorrentía superficial no alcanza valores notables a pesar de las elevadas precipitaciones. Los especímenes de T. pisuenensis sp. nov. fueron hallados junto a taludes arcilloso-margosos del hayedo. A menudo refugiados entre las pellas arcillosas desprendidas de los taludes que jalonan la ladera o entre los depósitos húmedos de hojarasca. Esta especie singular convive en la zona con otras especies de Trechus menos adaptadas a la vida hipogea como T. quadristriatus (Schrank, 1781) y T. barnevillei Pandellé, 1867. Estrechamente relacionado con el nicho que ocupa $T$. pisuenensis sp. nov., aparece de forma abundante Iberotrechus bolivari (Jeannel, 1913), paleoendemismo ibérico del que recientemente se ha descubierto su forma de vida epigea (Ortuño \& Toribio, en prensa). Además, se han observado otras especies de carábidos que son coincidentes en el espacio y en el tiempo con T. pisuenensis sp. nov., muchas de ellas con evidente vocación edáfica y nemoral, como por ejemplo: Carabus (Eucarabus) deyrollei Gory, 1839; Loricera pilicornis pilicornis (Fabricius, 1775); Notiophilus biguttatus (Fabricius, 1779); N. rufipes Curtis, 1829; Leistus (Leistus) barnevillei Chaudoir, 1867; Bembidion (Peryphanes) stephensii Crotch, 1869; B. (P.) deletum schulerianum Müller-Motzfeld, 1986; B. (Nepha) callosum subconnexum De Monte, 1953; Cryobius pumilio (Dejean, 1828); C. aralarensis (Mateu, 1945) у Pterostichus (Pterostichus) cristatus heydenianus Jacobson, 1907.

\section{Posición taxonómica y discusión}

Por los caracteres externos y la conformación del edeago, esta especie podía incluirse dentro del "grupo T. angusticollis" y considerarse cercana a ciertas especies de los montes cantábricos, con quienes coincide al tener los ángulos posteriores del pronoto más vivos y una evidente regresión ocular: T. pieltaini Jeannel, 1920; T. escalerai Abeille, 1903; T. apoduvalipenis Salgado \& Ortuño, 1998; T. marcilhaci Pham, 1987 y T. saxicola Putzeys, 1870 (la ssp. desbordesi A. Gaudin in Colas \& Gaudin, 1935). No obstante, T. pisuenensis sp. nov. se diferencia muy bien de las especies citadas por mostrar la base del pronoto muy ancha (en las demás especies la base del pronoto es más estrecha que el borde anterior), los hombros más conspicuos y las estrías de los élitros muy profun- 
das y fuertemente punteadas (como consecuencia las interestrías son más convexas). A pesar de los escasos caracteres morfológicos que T. pisuenensis sp. nov. tiene en común con estas especies ( $T$. saxicola, T. pieltaini, T. escalerai, T. apoduvalipenis y T. marcilhaci), sí parece más próxima a $T$. saxicola, de la que también se diferencia por la forma tan peculiar del pronoto, por el contorno de los élitros (anchos y subparalelos), por la conformación del lóbulo medio del edeago y la forma de la pieza del saco interno, y en general, por su mayor tamaño.

No obstante, la manifestación de la estriación elitral de T. pisuenensis sp. nov. entra en abierta contradicción con lo manifestado por Jeannel (1927) para poder pertenecer al "grupo T. angusticollis", el cual reúne especies con estrías finamente punteadas y las interestrías planas. Esta peculiaridad, sumada a otras (diente labial netamente escotado, pronoto amplio con la base ancha, élitros subparalelos, estría recurrente apical en contacto con la quinta estría y singular forma del edeago con una sola pieza en el saco interno), sitúa a $T$. pisuenensis sp. nov. en la vecindad del "grupo $T$. amplicollis", extraño a la fauna ibérica. En este caso, la especie nueva se desmarcaría de todas las del grupo por sus adaptaciones morfológicas a la vida hipogea (ver descripción) mientras que las demás, notoriamente epigeas, son aladas, macroftalmas (en general con ojos salientes) y fuertemente pigmentadas con reflejos irisados. Por lo expuesto, cabría esperar cierto parecido entre $T$. pisuenensis sp. nov. y la especie vecina geográfica del "grupo T. amplicollis", al menos en lo concerniente a caracteres internos (genitalia masculina y femenina, ver como ejemplo Ortuño, 2004) poco modificables por la presión del medio. En este sentido, los resultados dejan el problema en el punto de partida puesto que T. amplicollis Fairmaire, 1859, muestra los genitales masculinos y femeninos considerablemente diferentes de los de T. pisuenensis sp. nov., no pudiéndose confirmar la hipótesis de su filiación a este último grupo de especies.

Cabe la posibilidad de que T. pisuenensis sp. nov. pueda constituir un grupo nuevo, siguiendo los criterios de Jeannel (1927), si bien, a nuestro modo de ver se hace urgente una profunda revisión de estos grupos. Con el paso del tiempo se han descrito numerosos taxones nuevos, situación que ha trastocado la ciclópea sistemática del insigne entomólogo francés. Quizá sea oportuno plantear una futura revisión de los grupos incluyendo, como no puede ser de otro modo, nuevos criterios anatómicos, como por ejemplo la genitalia femenina (externa e interna).
En los últimos años, los hayedos se han revelado como refugio de Trechini hipogeos (Toribio \& Rodríguez, 1997; Carabajal et al., 1999; Toribio, 2001: 49; Ortuño \& Toribio, en prensa) en la Península Ibérica. Es bien conocida la propensión a la microendemicidad que muestran muchos Trechini debido a factores intrínsecos a este clado pero, en el caso de las especies ligadas a los hayedos, esta singularidad quizá se vea acrecentada por fenómenos de fragmentación del hábitat en pequeños bosques isla (Obeso \& García Manteca, 1990). Estas manchas discretas de bosque eurosiberiano, son el resultado de la regresión de una masa forestal continua que en los últimos miles de años se ha visto afectada por nuevas condiciones climáticas y, más recientemente, por acción antrópica (Ferreras \& Arozena, 1987: 108; Costa Tenorio et al., 1990; Obeso \& García Manteca, 1990). Estos "hayedos isla" merecerían más atención por parte de los biólogos pues son bolsas de biodiversidad que reúnen especies higrófilas, geófilas, lucífugas e hipogeas de extraordinario interés, muchas de ellas aún desconocidas para la Ciencia.

\section{Referencias}

Carabajal, E., García, J. \& Rodríguez, F., 1999. Descripción de un nuevo género y una nueva especie de Trechini (Coleoptera: Caraboidea: Trechidae) de la Cordillera Cantábrica. Elytron, 13: 123-131.

Casale, A., Vigna Taglianti, A. \& Juberthie, Ch., 1998. Coleoptera Carabidae. In: C. Juberthie \& V. Decu (eds.). Encyclopaedia Biospéologica. Vol. 2. Société de Biospéologie, Moulis-Bucarest: 10471081.

Colas, G. \& Gaudin, A., 1935. Sur de nouveaux Trechinae des Pyrénées occidentales. Revue Française d'Entomologie, 1(4): 245-253.

Costa Tenorio, M., García Antón, M., Morla Juaristi, C. \& SAinz Ollero, H., 1990. La evolución de los bosques de la Península Ibérica: una interpretación basada en datos paleobiogeográficos. Ecología, Fuera de Serie 1: 31-58.

Ferreras, C. \& Arozena, M. E., 1987. Guía física de España. 2. Los bosques. Alianza Editorial. Madrid. $394 \mathrm{pp}$.

Jeannel, R., 1927. Monographie des Trechinae. Morphologie comparée et distribution géographique d'un groupe de Coléoptères (II livraison). L'Abeille, 33: 1-592.

Obeso, J.R. \& García Manteca, P., 1990. Patrones de fragmentación del hábitat en el norte de España: el ejemplo de hayedos y robledales en Asturias. Ecología, Fuera de Serie 1: 511-520. 
ORTUÑO, V. M., 2004. An enigmatic cave-dwelling ground beetle: Trechus barratxinai Español, 1971 (Coleoptera, Carabidae, Trechinae, Trechini). Revue suisse de Zoologie, 111(3): 551-562.

Ortuño, V. M. \& TORIBIO, M., en prensa. Ecological relocation of the palaeoendemic Iberotrechus bolivari (Jeannel): from troglobiont to epigean (Coleoptera: Carabidae: Trechini). The Coleopterists Bulletin.

Salgado, J. M. \& OrtuÑo, V. M., 1998. The new cavedwelling beetle species (Coleoptera: Carabidae: Trechinae) of the Catabrian karst (Spain). The Coleopterists Bulletin, 52(4): 351-362.

Serrano, J., 2003. Catálogo de los Carabidae (Coleoptera) de la Península Ibérica. Monografías S.E.A., n. ${ }^{\circ}$ 9. Sociedad Entomológica Aragonesa. Zaragoza. $130 \mathrm{pp}$.
Toribio, M., 2001. Citas interesantes de Carabidae (Coleoptera) para la Península Ibérica ( $3^{\mathrm{a}}$ nota). Zapateri Revista aragonesa de entomología, 9: 49-52.

Toribio, M. \& Rodríguez, F., 1997. Un nuevo Trechus Clairville, 1806 de Cantabria, norte de España (Coleoptera: Carabidae: Trechinae). Zapateri Revista aragonesa de entomología , 7: 281-286.

Recibido, 29-III-2005

Aceptado, 14-VI-2005 Publicado, 14-VII-2005 